\title{
Seeding the Design Process for Future Problems
}

\author{
Peter Newman ${ }^{1}$, Stephen Forshaw ${ }^{2}$, Will Simm ${ }^{1}$, Maria Angela Ferrario ${ }^{3}$, \\ Jon Whittle ${ }^{1}$, and Adrian Friday ${ }^{1}$ \\ 1 School of Computing and Communications, Lancaster University \\ ${ }^{2}$ Lancaster Institute for the Contemporary Arts, Lancaster University \\ 3 Lancaster University Management School, Lancaster University \\ Lancaster LA1 4WA, UK \\ \{p.newman, s.forshaw, w.a.simm,m.a.ferrario, \\ j.n.whittle, a.friday\}@lancaster.ac.uk
}

\begin{abstract}
Designing with the community brings about a number of benefits, including tacit and contextual knowledge about the problem domain; this is especially apparent in rural settings. However, designing for problems that have yet to embed themselves in the fabric of society (i.e. future problems) poses a number of challenges, as they typically present intangible scenarios and concepts that have yet to be experienced by the wider-community. Using the OnSupply project as a case study, we share our experience in working with the Tiree community to address a future problem through a technology-mediated enquiry. Furthermore, we present a novel process that uses creative workshops augmented with physical artefacts to inform and learn from the community about a problem space, and to seed the design of a system that addresses it.
\end{abstract}

Keywords: User-driven, Creative workshop, Design, Future problem.

\section{Introduction}

Designing systems with the user community can often lead to the development of appropriate solutions, which is a consequence of the user being the expert of their own experience [1. Working with the community is even more crucial in rural settings, as systems are almost invariably tailored for urban users [2]. As such, it is important not to overlook rural communities when developing systems that may affect all segments of society, as there will often be different cultural, societal, and technological constraints as compared to an urban environment.

However, there are circumstances where the intention is to design a system to address a problem that has yet to establish itself in the fabric of society (i.e. future problems). As these problems often describe intangible scenarios and concepts that have yet to be experienced by the wider-community, designing in a bottom-up, user-centric manner can pose a number of challenges:

- Lack of domain expertise. The involvement of users is often intended to draw upon their expert knowledge of the current system (i.e. domain experts); however, designing in a community-driven manner for an unfamiliar domain potentially means that the traditional level of expertise is not present. 
- Lack of visibility/tangibility. As future problems often describe emergent, abstract problems, they are typically less visible to the greater society, meaning that the users must first be convinced of its existence and importance before any fruitful dialogue may occur.

In this paper, we describe our experience of using creative workshops augmented with physical artefacts to help support the discussion and design of a system that addresses a future problem. This is done by gradually immersing the intended end-users into the problem context through the use of game-based scenarios and physical artefacts. We present OnSupply as a case study, which describes a possible future scenario where the supply of power cannot meet the current pattern of demand placed upon the grid.

\section{Designing the Future}

The development of systems and strategies to address future problems (i.e. scenarios) can be seen in a number of domains, including air traffic control and power generation 34. However, there are few research initiatives that investigate the design and planning of such systems in a community-driven context. Instead, the majority of work focuses on design with expert users.

The use of media as a means to help vision future technology is sometimes used to help design for the future. Macini et al. present ContraVision [5], which demonstrates a fictional future technology to users through a video that uses both positive and negative scenarios to elicit reactions from the user. Another example of using scenarios and users to vision future technologies is seen in the works of Go et al. and Carroll et al. 67, who both present novel participatory design approaches for the design of future systems (i.e. mobile phone and virtual learning environment). However, whilst these approaches attempt to elicit the design constraints and requirements of systems for the future, they typically involve the use of domain experts to elicit design considerations.

\section{Supporting the Creative Process}

There are a number of methods that have been explored to support discussion in a workshop setting. One well known method is that of Six Thinking Hats, which was presented by De Bono [8] in 1985, and describes a way of assigning a role to play for each participant. Creativity is another dimension to consider in a workshop setting, and can often be encouraged by the presence of physical artefacts. Indeed, physical artefacts often play a crucial role in design, a sentiment shared by Hansen and Dalsgaard [9. Maiden et al. [10] present one such method, which aids the creative process through the use of physical artefacts and lucid workshops. In a similar vein, Vyas et al. attempt to support the sharing of artefacts and related notes through a mobile application called CAM [1]. Although this demonstrates that physical artefacts have been applied to design in the computing domain, there is a lack of examples showing the use of physical artefacts to design in a non-expert setting. 


\section{Design Using Physical Artefacts}

To address the challenges described in Section 11, we present a process that uses physical artefacts, scenario-based games, and creative workshop sessions to teach, inform, and learn from the participants about the problem space. Through this process and by involving them in the design of the end system, we aim to encourage and maintain interest from the community, and foster the creation of proxy domain experts within the pool of participants.

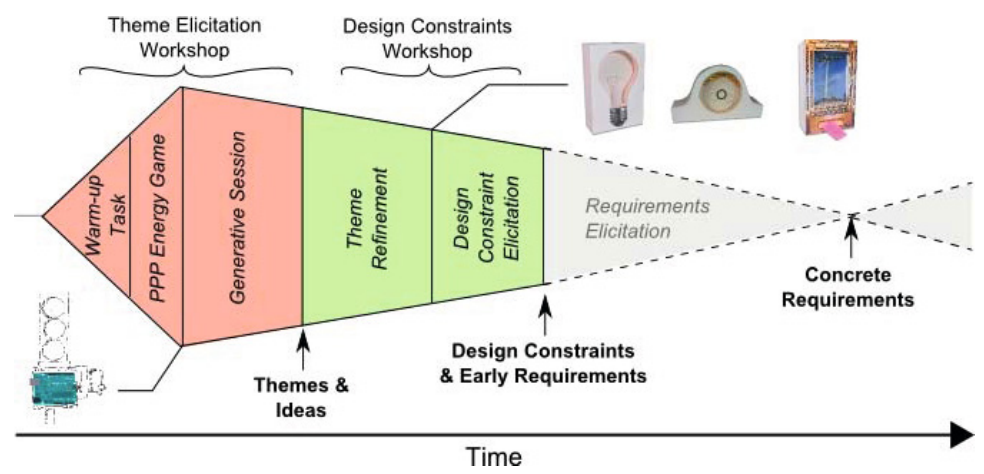

Fig. 1. Overview of the process used to elicit themes and design constraints

This process is embodied by two phases, which are illustrated in Figure1 The purpose of two workshops is to give the facilitators time to realise the elicited themes from the first workshop as low fidelity physical artefacts, which we would subsequently present back to the community. The use of physical artefacts and game-based scenarios helps to mitigate the lack of visibility/tangibility of a future problem by allowing the participants to physically engage with the problem context. This provides an element of learning through experience and concrete example, which are important parts of the learning cycle [12].

\section{Case Study : OnSupply}

Recently, there has been a gradual shift in the UK from conventional coal-fired power stations to less carbon-intensive methods of power generation, such as renewable (e.g. wind turbine, photovoltaic etc.) energy [13. However, as renewable energy is often time and weather dependent, the output of these low carbon alternatives can shift quickly, leading to a highly variable supply of electricity. Further compounding this problem is the gap between the decommissioning of current, older-generation power stations, and the commissioning and construction of new ones 14. The Catalyst project 15] OnSupply is working with the island community of Tiree in a bottom-up approach to realise the future systems needed to help communities adapt to a highly variable energy supply. 


\subsection{Overview}

We held two 90 minute workshop sessions on Tiree in an attempt to elicit design constraints for a digital intervention from the island community. Through an open invitation in the local newsletter, we attracted participants from a wide variety of different backgrounds, including the local school headteacher, an electrical engineer, a local artist, and older teenagers.

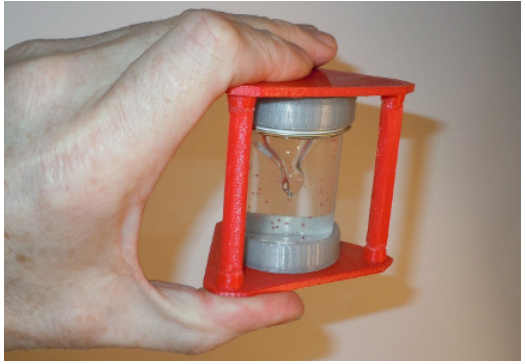

(a) 3D printed Inter-dimensional Energy prop

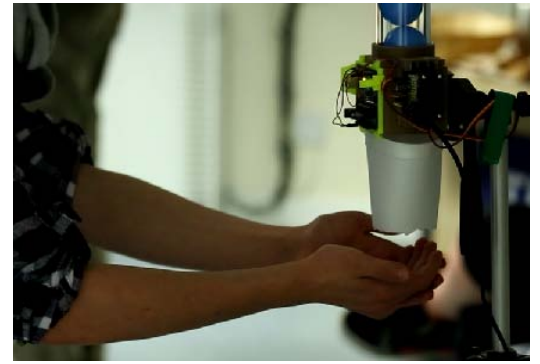

(b) Ping pong ball dispenser from scenario-based game

Fig. 2. Physical artefacts used during the first workshop

\subsection{Theme Elicitation Workshop}

The first workshop was intended to engage with the adults and teenagers on the island, encourage ideation around the utilisation of a variable energy supply, and help position the participants within the described future problem space. The following describes the tasks within the first workshop.

- Warm-up task. The main purpose of the warm-up task was to break down any barriers between the participants and the facilitators. Borrowing from the Neutral-Zone Metaphor technique [16], Inter-dimensional Energy was presented to the participants as a fictional, alien power source that suffered from intermittent black-outs - akin to the island's power infrastructure. It was represented as a small glass vial enclosed in a 3D printed plastic manifold (see Figure 2a), and the participants were asked to imagine ways that they may harness it as an energy source.

- Scenario-based game. As supply-driven energy use is not the currently established model of energy consumption, a way was needed to present it in a manner that was both understandable and interesting to different backgrounds. In order to achieve this, the Ping Pong Power (PPP) game was developed to help the participants better visualise variable energy supply. The aim of the game involved the participants needing to power a number of household appliances using dispensed ping pong balls (energy) (see Figure $2 \mathrm{~b}$ ). To gradually ease participants into the problem context, rules were slowly introduced to the game to mimic the properties of variable supply. 
- Theme generation task. The aim of the generative task was to give the participants of the workshop a chance to reflect on the previously played PPP game. Because the game and OnSupply are so closely linked, we expected that any ideas generated would encompass both real-world and in-game mechanisms to help better utilise energy/balls. During this task, the participants were encouraged to write down any ideas they had.

Theme Elicitation Results. In total, 16 participants attended the workshop, with an age range of 16-60. During the warm-up session, approximately 40 ideas for utilising the fictional inter-dimensional energy were suggested, with ideas ranging from charge a battery to cook[ing] popcorn. Although these ideas had no bearing on those generated in the generative task, they played an important role in encouraging the participants to engage with each other - in part spurred on by witnessing lighthearted and out-worldly ideas being presented by the rest of the group. Furthermore, the PPP game and its physical artefacts (ping pong ball dispensers) appeared to generate excitement, as evidenced by exclamations of "Catch it!" and "Come on, come on!" in reference to the dispensed balls, and laughter and excitement being witnessed throughout the task. Finally, the generative task resulted in a number of themes that suggest the participants were interested in ways to store, forecast, and predict generated electricity; these themes are brought forward in the next workshop.

\subsection{Design Constraints Workshop}

The second workshop was intended to build upon the themes elicited in the first workshop, and to refine them into a set of design constraints. As this workshop relied on the participants to understand the problem context, they were selected from the pool of people who attended the first workshop, and from other previous engagements with the community. The workshop consisted of the two elements: Theme refinement and Design constraint elicitation.

- Theme refinement. This part involved a brief overview of the themes (and thus artefacts) generated by the previous workshop, which had since been embodied in a number of low fidelity physical artefacts (or sketches). The design of these sketches was informed by our notes of the island, and designer instinct; in total, 5 sketches were made (see Figure 3), and they were distributed around a table central to the participants.

During their development, it was important to consider the perception the participants had towards the sketches, and if they would feel free to draw on, alter, and potentially discard them. As such, they were made from low fidelity materials (e.g. craft foam) as a way to decrease their perceived value.

- Design constraint elicitation. The final part of the second workshop included a short period to reflect upon any new themes and comments placed on the table by the participants. During this latter half of the session, the participants were prompted to list the desirable (and undesirable) features of any 
future system that may be used to address the proposed problem. Once the session had ended, the participants were invited to present to the rest of the group and provide an explanation of the constraints they wrote, and why they were important to them. This would give the rest of the group an opportunity to raise any issues or conflicting viewpoints.
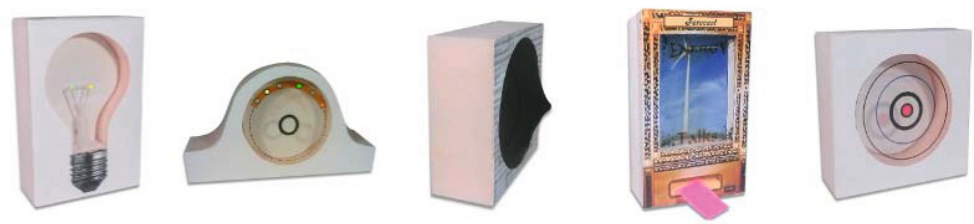

Fig. 3. Number of physical sketches presented to participants. (Left to Right) : Lightbulb Sketch, Clock Sketch, Haptic Sketch, Zoltar Sketch, and Target Sketch

Design Constraints Results. In total, 8 participants were involved in the Design constraints workshop, the majority of which were invited back from the previous workshop; these participants were invited back based on their continued correspondence with us. From the initial themes brought forward from the first workshop, over 60 individual design artefacts were generated by the participants, including sketches and comments drawn on the main worksheet.

The low fidelity sketches played an important roll in the seeding of this discussion, which is demonstrated by the participants often picking up, playing with, and annotating the sketches - akin to show and tell. Further evidence of the sketches being used to inspire ideation can be seen in comments written on the table worksheet, such as "clock = great, [needs] forecasting in real-time" and "It should have tactile feedback for the sight/hearing impaired"; the latter comment was made in context of the Haptic sketch. These comments were predominantly placed in close proximity to the physical sketches.

\section{Reflections}

The workshops seemed to be well received by the island community, a sentiment echoed by one of the participants _ " [...] I think for me, this whole thing shows the importance of how productive collaboration can be, and how it can facilitate this kind of brainstorming session [...]". As a vehicle to engage with the community, the workshops were equally successful as a means to convey the properties and concepts of a future problem (i.e. intermittent and variable energy supply). This was evident in the understanding of the problem context shown by the participants after the warm-up tasks and during the second workshop. 
As noted during the session, there was also evidence to suggest that the sketches were used to help inspire ideation and acted as talking points between the participants; indeed, participants would often gesticulate with the sketches to convey an idea. We would go further to suggest that the presentation of themes and physical sketches also helped to focus the design artefacts being generated by providing a focal point for the participants to discuss.

\subsection{Lessons Learnt}

From these workshops, we have learnt a number of valuable lessons that may help other creative workshops; these lessons are:

- Physical over abstract. During our first workshop, we found that the verbal description of a concept (such as OnSupply) wasn't sufficient for a participant to fully understand and visualise it. Our experience shows that using physical props is very useful in conveying ideas to and between the participants.

- Allow time for reflection. There was the temptation to hold both workshop sessions as one larger session; however, due to the time constraints of the participants, we decided to split the workshop into two. Consequently, we were able to spend more time analysing the results from the first part of the process, and develop higher fidelity sketches. Furthermore, we believe this gave the participants more time to reflect on the first workshop.

- Know the context. An important factor to the success of these workshops has been the other engagement opportunities that surround it. Through interviews and other ethnographic efforts, we feel we have gained a level of understanding of the island context, which has better equipped us to analyse the data we have gathered. As such, we urge the reader to get to know the culture of the workshop participants to better design around and with them.

\section{Conclusion}

Designing with a community plays an important role in the development of ICT systems, as it allows for the development of appropriate solutions to the problems identified by the community. However, designing for future problems in a user-centred manner can be challenging, especially as these problems provide a context that may be unfamiliar to those involved.

This paper has presented our experience of eliciting design constraints for a future problem through the use of playful, creative workshops in a user-centred manner using physical props and scenario-based games. Through these creative workshops, we have been able to facilitate an ongoing dialogue with the Tiree community, whilst distilling and disseminating the properties of the energy scenario. Although the results of this work are promising, we feel that further evaluation to fully gauge the efficacy of this approach is needed.

The future direction of this work includes revisiting Tiree with early prototypes inspired and guided by the design constraints elicited during the workshops described herein. We also hope to continue with the workshop participants as lead users by involving them in iterations of these prototypes. 


\section{References}

1. Sanders, E.B.N., Stappers, P.J.: Co-creation and the new landscapes of design. Co-design 4(1), 5-18 (2008)

2. Siew, S.-T., Yeo, A.W.: Employing participatory action research to augment software development for rural communities. In: Proc. of the 25th BCS Conference on Human-Computer Interaction, BCS-HCI 2011, pp. 171-176. British Computer Society, Swinton (2011)

3. Hunter, G., Ramamoorthy, K.: National airspace strategies for future demand scenarios in inclement weather. In: The 24th Digital Avionics Systems Conference, DASC 2005, vol. 1 ( October 2005) 3.E.2-31-12

4. Widen, J.: Correlations between large-scale solar and wind power in a future scenario for sweden. IEEE Transactions on Sustainable Energy 2(2), 177-184 (2011)

5. Mancini, C., Rogers, Y., Bandara, A.K., Coe, T., Jedrzejczyk, L., Joinson, A.N., Price, B.A., Thomas, K., Nuseibeh, B.: Contravision: Exploring users' reactions to futuristic technology. In: Proc. of the SIGCHI Conference on Human Factors in Computing Systems, CHI 2010, pp. 153-162. ACM, New York (2010)

6. Carroll, J., Rosson, M., Chin, G., Koenemann, J.: Requirements development in scenario-based design. IEEE Transactions on Software Engineering 24(12), 1156-1170 (1998)

7. Go, K., Takamoto, Y., Carroll, J.: Designing a mobile phone of the future: requirements elicitation using photo essays and scenarios. In: 18th International Conference on Advanced Information Networking and Applications, AINA 2004, vol. 2, pp. 475-480 (March 2004)

8. De Bono, E.: Six Thinking Hats. Little, Brown Book Group Limited (1985)

9. Hansen, N.B., Dalsgaard, P.: The productive role of material design artefacts in participatory design events. In: Proceedings of the 7th Nordic Conference on Human-Computer Interaction: Making Sense Through Design, NordiCHI 2012, pp. 665-674. ACM, New York (2012)

10. Maiden, N., Jones, S.: The rescue requirements engineering process: An integrated user-centred requirements engineering process, version 4.1. Technical report (February 2004)

11. Vyas, D., Nijholt, A., van der Veer, G.: Supporting cooperative design through "living" artefacts. In: Proceedings of the 6th Nordic Conference on Human-Computer Interaction: Extending Boundaries, NordiCHI 2010, pp. 541-550. ACM, New York (2010)

12. Kolb, D.: Experiential learning: experience as the source of learning and development. Prentice-Hall (1984)

13. UK Government: UK Renewable Energy Roadmap Update 2013 (2014)

14. EDF Energy: The Energy Gap (2014), http://www.edfenergy.com/energyfuture/key-info/the-energy-gap

15. Whittle, J., Ochu, E., Ferrario, M.A., Southern, J., McNally, R.: Beyond Research in the Wild: Citizen-Led Research as a Model for Innovation in the Digital Economy. In: Proc. Digital Futures (2012)

16. Forshaw, S., Cruickshank, L., Dix, A.: Collaborative Communication Tools for Designing: Physical-Cyber Environments. In: Proc. of HCI 2012 - The 26th BCS Conference on Human Computer Interaction (2012) 